\title{
In Vitro Efficacy of Cefepime Against Multi-Drug Resistant Pseudomonas aeruginosa - An Alarming Situation in our Setup
}

\author{
Luqman Satti ${ }^{*}$, , Shahid Abbasi ${ }^{2}$, Tanveer Ahmed Qumar ${ }^{1}$, Muhammad Shoaib Khan ${ }^{3}$ and \\ Zahid Ahmed Hashmi ${ }^{4}$ \\ ${ }^{I}$ Department of Pathology, Combined Military Hospital, Dera Ismail Khan, Pakistan \\ ${ }^{2}$ Consultant Microbiologist, Armed Forces Institute of Pathology, Rawalpindi, Pakistan \\ ${ }^{3}$ Department of Surgery, Combined Military Hospital, Dera Ismail Khan, Pakistan \\ ${ }^{4}$ Gomal Medical College, Dera Ismail Khan, Pakistan
}

\begin{abstract}
Background: Third generation cephalosporins are still most commonly used antibiotics empirically in our setup leading to emergence of resistance in this group. In this environment of increasing resistance, empirical use of this group of antibiotics would be a futile effort. Therefore, this study was conducted to evaluate the in vitro efficacy of fourth generation cephalosporin cefepime against multi-drug resistant isolates of Pseudomonas aeruginosa from various clinical specimens in our setup.

Place and Duration of Study: Department of Pathology, Combined Military Hospital Dera Ismail Khan, Pakistan from January 2011 to May 2011.

Methodology: All the clinical specimens giving growth of Pseudomonas aeruginosa were included in the study. Modified Kirby-Bauer disk diffusion method was used for sensitivity testing. Isolates resistant to three or more than three groups of antibiotics were included in the final results.

Results: A total of 100 multi-drug resistant Pseudomonas aeruginosa isolates were cultured from various clinical specimens. Out of 100 isolates, $71 \%$ were resistant to cefepime while resistance rate of amikacin, gentamicin, ceftazidime, tazobactem/piperacillin, sulbactam/piperacillin, imipenem and polymyxin were $61 \%, 83 \%, 72 \%, 10 \%, 2 \%, 45 \%$ and $2 \%$ respectively.

Conclusion: Drug resistance in Pseudomonas aeruginosa is alarming as it is now increasing even in newer generation of drugs. It is the need of hour to follow strict hospital infection control and antibiotic policies to halt the spread of this resistance.
\end{abstract}

Keywords: Cefepime, multi-drug resistance, Pseudomonas aeruginosa.

\section{INTRODUCTION}

Pseudomonas aeruginosa is an oxidase positive, nonfermenter and produces visible pigments, characteristics which are used for its rapid diagnosis in the laboratory. It is also a part of normal skin flora of humans but can cause life threatening opportunistic infections specially in immunocompromised hosts [1]. It has been implicated in diverse group of infections such as otitis externa, burn wounds, decubitus ulcers, cystic fibrosis, urinary tract infections, ventilator associated pneumonia (VAP) and septicemia specially in neutropenic and transplant patients. It has posed a serious problem in nosocomial infections specially in intensive care units (ICUs) because of its innate resistance to many antibiotics [2]. It has also been involved in biofilm formation particularly in cystic fibrosis patients with its unique characteristic to develop resistance to all

*Address correspondence to this author at the Department of Pathology, Combined Military Hospital, Dera Ismail Khan, Pakistan;

Tel: +923215570838; E-mail: Luqmansatti@hotmail.com antibiotics with prolonged therapy [3]. In recent years, multidrug resistant (MDR) Pseudomonas aeruginosa specially in burn wounds and decubitus ulcers has emerged rapidly creating serious problems for the clinicians [4].

About $50 \%$ of the antibiotics prescribed are irrational (wrong dose or duration, antibiotics in viral infections) leading to the emergence of resistant isolates [5]. With the extensive use of third generation cephalosporins (most widely prescribed antibiotics) in hospitals, now many isolates are resistant to even this class of antibiotics [6]. Cefepime is the most active fourth generation cephalosporin which was introduced in 1993. It has rapid penetration into the periplasmic space as compared to third generation cephalosporins and has more activity against gram positive and gram negative organisms. It has remarkable activity against MDR isolates of Pseudomonas aeruginosa, Klebsiella pneumoniae, Citrobacter spp, Serratia spp, Proteus mirabilis but is less active against Bacillus fragillis [4]. It is ineffective against methicillin-resistant strains of Staphylococcus and Enterococcus spp. It can be used in the 
management of pneumonia, skin and soft tissue infections, urinary tract infection and neutropenic patients. It is the best empirical choice for the treatment of hospital acquired infections specially in intensive care units [7]. Because of its stability against many beta-lactamases and being a poor inducer of AmpC beta-lactamases, it can be used against those Enterobacteriaceae which are resistant to third generation cephalosporins.

Very few studies have been reported on the evaluation of cefepime against MDR Pseudomonas aeruginosa. In Pakistan, to date, no study has been published on the evaluation of cefepime agaisnt MDR Pseudomonas aeruginosa. In the background of increasing resistance pattern, excessive empirical use of third generation cephalosporins and poor hospital infection control policies in our set-up, this study was conducted to see the efficacy of cefepime against MDR Pseudomonas aeruginosa from various clinical specimens.

\section{MATERIALS AND METHODOLOGY}

This laboratory based descriptive study was carried out in the department of Pathology, Combined Military Hospital Dera Ismail (D.I.) Khan, Pakistan. A total of 100 MDR Pseudomonas aeruginosa isolates from various clinical specimens were included in the five months study period from January 2011 to May 2011. This hospital receives specimens for bacterial culture mainly from District Hospital, and Mufti Mehmood Teaching Hospital D.I. Khan. Specimens from the patients admitted in Combined Military Hospital D.I. Khan were also included in our study. Pus/pus swab specimens were mostly from burn patients, wound infections, and diabetic foot ulcers. Ear swabs were from patients suffering from chronic suppurative otitis media (CSOM). All the sputum specimens were from patients suffering from COPD while paired blood specimen was collected for blood culture. Repeat or multiple specimens from same patients giving growth of Pseudomonas aeruginosa were excluded from the study.

\section{Inoculation and Culture}

In the protocol followed, all the clinical specimens (pus, pus swab, urine, sputum, blood, and tissue) were inoculated on 5\% Sheep Blood Agar (Oxoid, UK), MacConkey agar (Oxoid, UK) Chocolate Agar (Oxoid, UK) and incubated at $37^{\circ} \mathrm{C}$ for 24-48 hours. Blood culture specimens were incubated in Brain Heart Infusion (BHI) broth (Oxoid, UK) and subcultured on every alternate day for upto seven days. Urine samples were inoculated on CLED (Oxoid, UK) medium and incubated at $37^{\circ} \mathrm{C}$ for 24 hours. Gram stain and rapid oxidase test was performed on all the cultured isolates for the confirmation of Pseudomonas aeruginosa.

\section{Antimicrobial Susceptibility Testing (AST)}

Modified Kirby-Bauer disk diffusion method was used for sensitivity testing and the disks employed were cefepime $30 \mu \mathrm{g}$ (Oxoid, UK), amikacin 30 $\mathrm{g}$ (Oxoid, UK), gentamicin $10 \mu \mathrm{g}$ (SPAN Diagnostic, France), ceftazidime $30 \mu \mathrm{g}$ (Oxoid, UK), tazobactem/piperacillin 10/100 $\mu \mathrm{g}$ (Oxoid, UK), sulbac-

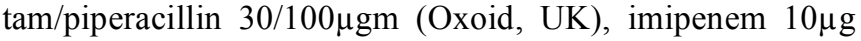

(Oxoid, UK) and polymyxin B 300units (Oxoid, UK). All the sensitivity plates were incubated at $37^{\circ} \mathrm{C}$ for $16-18$ hours. Pseudomonas aeruginosa ATCC 25853 was used as control to check disks potency.

\section{Interpretation}

After incubation period, zone diameters around the disks were measured with a scale in millimeters and each zone size was compared with known standards [8]. The isolate was then labelled as sensitive, resistant or intermediate depending upon the zone size. A Pseudomonas aeruginosa resistant to three or more group of antibiotics was considered as MDR. Antibiotics giving zone sizes in the intermediate ranges are usually not effective against that isolate, however, increased dose of that antibiotic can be given if there are no other therapeutic options [9].

\section{RESULTS}

Out of total 100 specimens yielding growth of Pseudomonas aeruginosa, 63 (63\%) were from male patients and 37 (37\%) from female patients. The age range was 4-72 years with mean age $38 \pm 15.2$ years with maximum number of patients in the fourth decade of life. Maximum number of isolates were recovered from pus/pus swabs followed by ear swabs as shown in Fig. (1). Pseudomonas aeruginosa from pus specimens showed maximum resistance to cefepime as shown in Table $\mathbf{1}$.

Most effective drug in our study against Pseudomonas aeruginosa was sulbactam/piperacillin followed by tazobactem/piperacillin. Comparison of sensitivity pattern of cefepime with other antibiotics is shown in Table 2. Majority of the isolates were resistant to five antibiotics as depicted in Fig. (2). There were two isolates from pus which were even resistant to polymyxin that is pan-resistant Fig. (3).

\section{DISCUSSION}

Betalactamase production and use of multiple efflux pumps specially MexAB-OprM efflux system are the main mechanisms of resistance in Pseudomonas aeruginosa [10]. In recent years, methicillin-resistant Staphylococcus aureus, a so called "Superburg" casuing noscomial infections and multidrug resistant Pseudomonas aeruginosa infections have gained much attention among health care professionals [11]. It is evident from our study that Pseudomonas aeruginosa is becoming more resistant to even newer generation cephalosporins such as cefepime. In our study, overall resistance of cefepime was $71 \%$ and pus specimens yielded majority of the resistant isolates. Sulbactam/piperacillin and tazobactem/piperacillin were the most effective antibiotics against Pseudomonas aeruginosa in our study with $98 \%$ and $92 \%$ susceptibility respectively. Sensitivity of imipenem was $55 \%$ while gentamicin was the least sensitive drug in our study that is only $17 \%$ isolates were sensitive. As far as in vitro efficacy of cefepime is concerned, our results are comparable to the findings of a study done by Jazani et al. in which resistance rate of cefepime against Pseudomonas aeruginosa was $75.4 \%$ but imipenem was most effective drug with resistance rate of only $27 \%$ [4]. In another study done by Gad et al. on 81 clinical isolates of Pseudomonas 


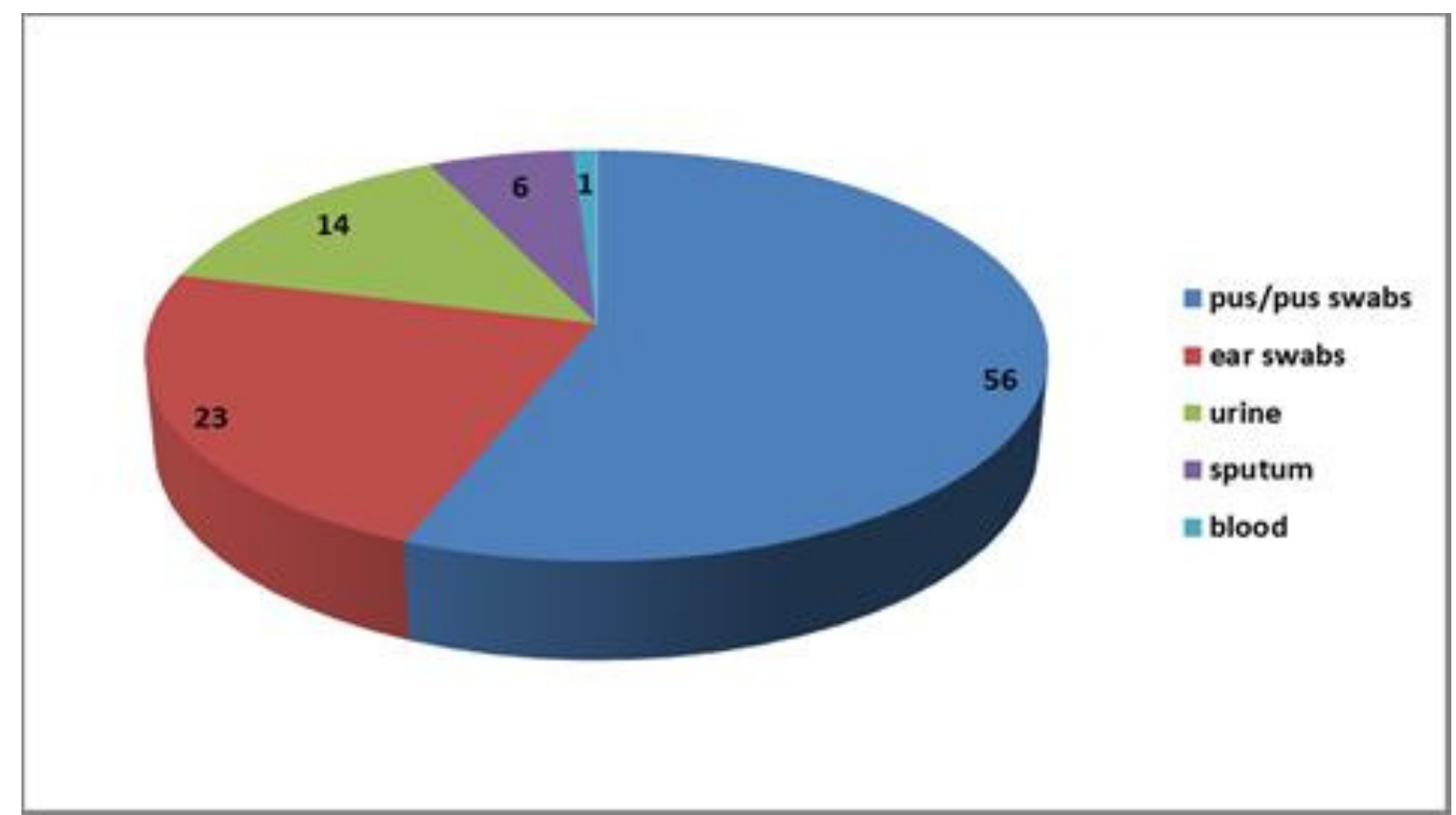

Fig. (1). Frequency of isolation of Pseudomonas aeruginosa from various clinical specimens ( $\mathrm{n}=100)$.

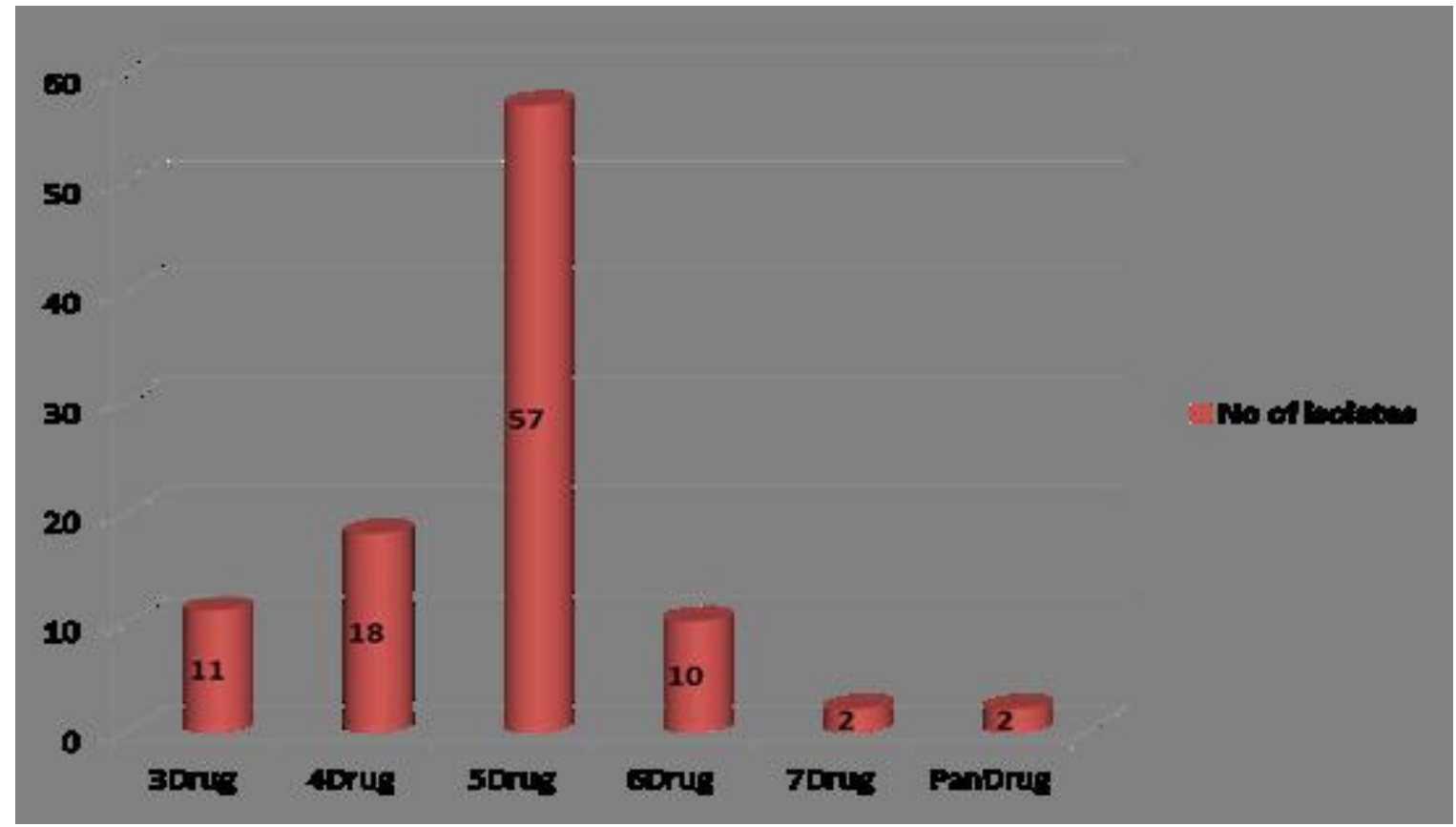

Fig. (2). Analysis of drug resistance pattern against Pseudomonas aeruginosa $(\mathrm{n}=100)$.

aeruginosa, resitance rate of cefepime was $29 \%$ while amikacin was the most effective drug (resistance rate $8 \%$ ) followed by meropenem (22\%) [12]. In another study done by de Macedo and Santos, imipenem and amikacin were the most useful antibiotics against Pseudomonas aeruginosa which is not in line with our results [13].

Second to pus specimens, ear swabs were the most common specimens submitted in our laboratory. All the ear swabs yielding growth of Pseudomonas aeruginosa were from patients suffering from chronic suppurative otitis media (CSOM). Out of total 23 isolates of Pseudomonas aeruginosa from CSOM patients 10 (43.5\%) were sensitive to cefepime while again sulbactam/piperacillin and tazobactem/piperacillin were most effective drugs. Similarly out of 14 urine specimens, cefepime was effective in 8 $(57.1 \%)$ isolates. In a study done by Patel et al., out of total 64 urinary isolates of Pseudomonas aeruginosa, resistance rate of cefepime was $15.6 \%$ [14].

In our study, there were 5 specimens from burn patients in which Proteus mirabilis was also cultured along with Pseudomonas aeruginosa and all of them were also resistant to cefepime along with Pseudomonas aeruginosa. There was only one isolate of Pseudomonas aeruginosa which was isolated from paired blood specimen and the isolate was 
sensitive to cefepime. The patient was later on diagnosed as a case of chronic myeloid leukemia. Out of a total of 6 sputum specimens from patients with chronic obstructive pulmonary disease, only $2(33.3 \%)$ isolates were sensitive to cefepime while all were sensitive to tazobactem/piperacillin and three were resistant to imipenem. These findings are again in contrary to the findings of a study done by Christieson et al. in which out of total 67 Pseudomonas aeruginosa isolates from cystic fibrosis patients, $92.5 \%$ were sensitive to imipenem while $77.6 \%$ were sensitive to cefepime [15]. An interesting observation in our study was that $57 \%$ of the total isolates were only sensitive to sulbactam/piperacillin, tazobactem/piperacillin and polymyxin (5-drug resistant). This is an alarming situation as these three drugs are injectables, costly, and not easily available in remote settings like ours. The patient had to face many difficulties in receiving these drugs from larger cities or they are ultimately referred to tertiary care hospitals.

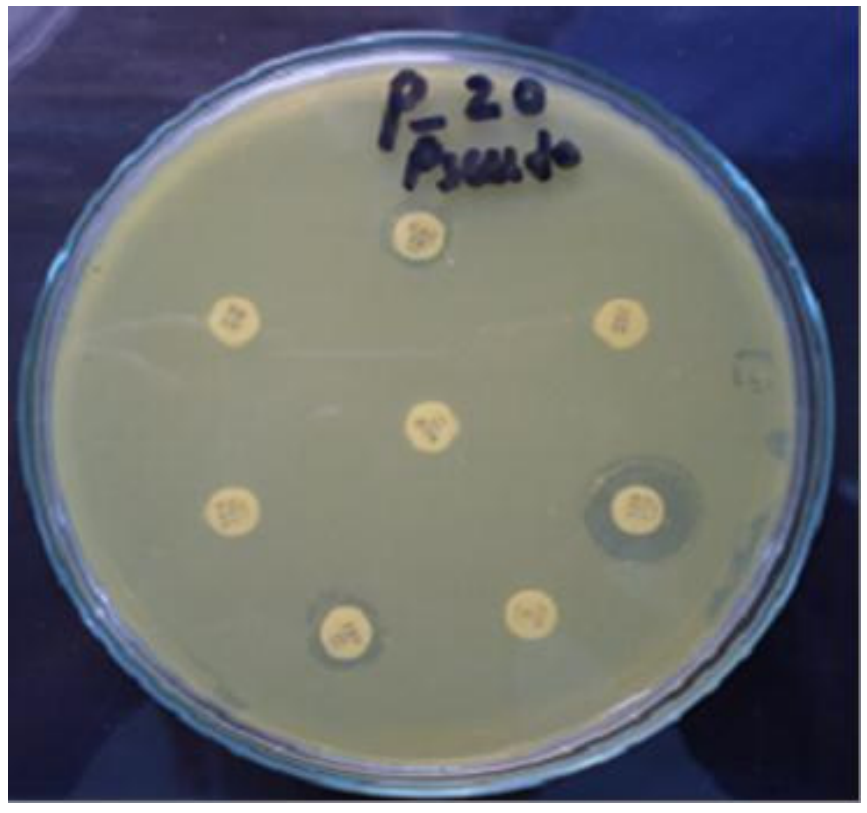

Fig. (3). A pan-drug resistant isolate of Pseudomonas aeruginosa from a burn patient.
Polymyxin B and colistin are older antipseudomonal drugs which have re-emerged in the settings of increased drug resistance [16]. They are now reserved as last option in treating MDR Pseudomonas aeruginosa. In our study, there were 2 isolates from burn patients which were even resistant to polymyxin (pan-drug resistant). Pandrug resistant isolates are a major concern for the clinicians as the therapeutic options are limited and presently the only choice left is polymyxin. Polymyxin has been used with success in cases of meningitis and pneumonia, but has been associated with renal toxicity [17]. The sensitivity pattern in our study shows that the resistance to even polymyxin is increasing adding to the miseries of patients.

Table. 1. Sensitivity Pattern of Pseudomonas aeruginosa Against Cefepime Isolated from Various Clinical Specimens $(n=100)$

\begin{tabular}{|c|c|c|c|}
\hline Specimen Type & Total Isolates & Sensitive & Resistant \\
\hline \hline Ear swab & 23 & 10 & 13 \\
\hline Pus & 56 & 08 & 48 \\
\hline urine & 14 & 8 & 6 \\
\hline sputum & 6 & 2 & 4 \\
\hline blood & 1 & 1 & - \\
\hline Total & 100 & $29(29 \%)$ & $71(71 \%)$ \\
\hline
\end{tabular}

\section{CONCLUSION}

Our study concludes that Pseudomonas aeruginosa, a lethal pathogen in hospital environment specially in burns and ICU patients is becoming resistant even to newer antibiotics such as cefepime. It is not only creating a grave challenge for the clinicians but is also very difficult to eradicate from the hospital environment. The main reasons for this critical situation are irrational use of antibiotics, poor hospital infection control policies, substandard drugs and lack of knowledge of organism's innate resistance to antibiotics. If timely interventions/measures are not taken to

Table. 2. Comparison of Sensitivity Pattern of Cefepime Against Pseudomonas aeruginosa with Other Antibiotics (n=100)

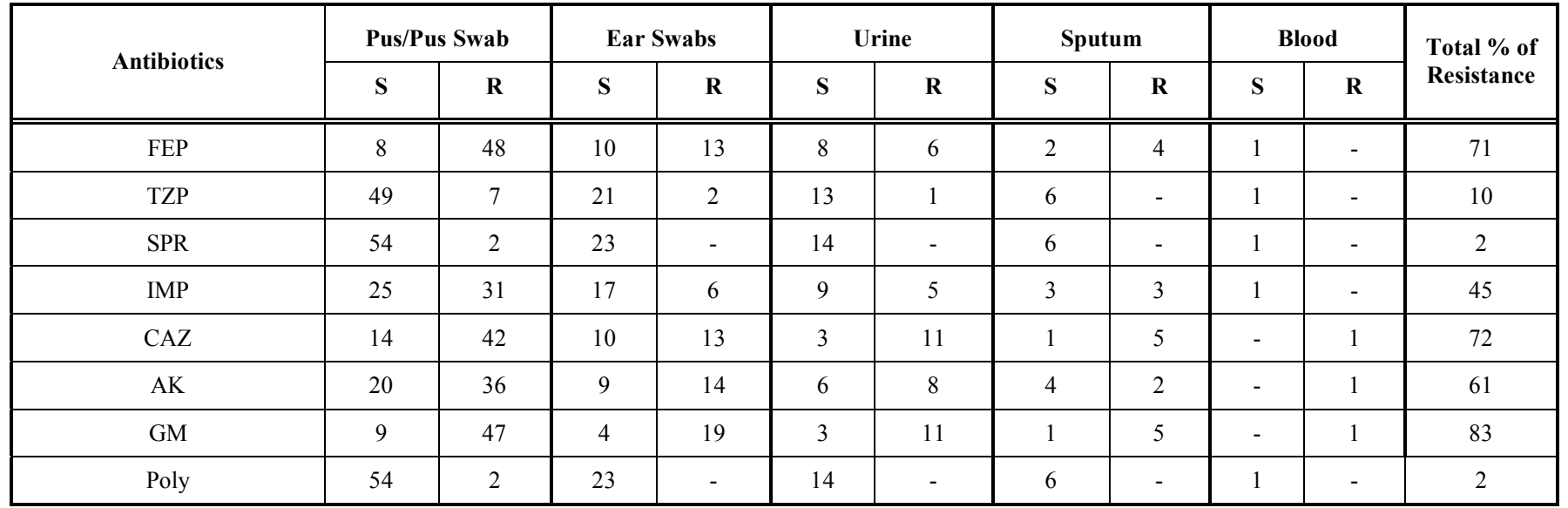

$\mathrm{S}=$ sensitive, $\mathrm{R}=$ resistant, $\mathrm{FEP}=$ cefepime, $\mathrm{TZP}=$ tazobactem/piperacillin, $\mathrm{SPR}=$ sulbactam/piperacillin, $\mathrm{IMP}=$ imipenem, $\mathrm{CAZ}=$ ceftazidime, $\mathrm{AK}=$ amikacin, $\mathrm{GM}=$ gentamicin, Poly = polymyxin 
prevent further resistance, then we may have to say that we are nearing towards the end of antibiotic era.

\section{REFERENCES}

[1] Larson, L. L.; Ramphal, R. Extended-spectrum beta-lactamases. Semin. Respir. Infect., 2002, 17, 189.

[2] Mesaros, N.; Nordmann, P.; Plesiat, P.; Roussel-Delvallez, M.; Van Eldere, J.; Glupczynski, Y. Pseudomonas aeruginosa: resistance and therapeutic options at the turn of the new millenium. Clin. Microbiol. Infect., 2007, 13, 560.

[3] National Committee for Clinical Laboratory Standards (NCCLS). Performance standards for antimicrobial disk susceptibility tests; approved standard, $7^{\text {th }}$ ed., 2000, Vol. 17.

[4] Jazani, N. H.; Babazadeh, H.; Sabah, Z.; Zartoshti, M. The evaluation of antibiotic resistance to cefepime in hospital isolates of Pseudomonas aeruginosa. J. Med. Biomed. Sci., 2010, 9, 17.

[5] Finch, R. Bacterial resistance to antibiotics. In: Denyer, S.P.; Hodges, N.A.; Sean, P.; Eds.; Hugo and Russell's Pharmaceutical Microbiology. $7^{\text {th }}$ ed., USA: Blackwell Science, 2004, p. 223.

[6] Zehra, A.; Naqvi, B. S.; Bushra, R.; Ali, S. Q. Comparative study on resistance pattern of different pathogens against Cefixime and Cefepime. J. Pharm. Sci., 2010, 3, 145.

[7] Endimiani, F.; Perez, F.; Bonomo, R. A. Cefepime: a reappraisal in an era of increasing antimicrobial resistance. Expert Rev. Anti. Infect. Ther., 2008, 6, 805.

[8] NCCLS. Performance standards for antimicrobial disk susceptibility tests; Approved Standard, 2000, $7^{\text {th }}$ ed., Vol. 17.

[9] Schwalbe, R.; Steele-Moore, L.; Goodwi, A. C. Antimicrobial susceptibility testing protocols. Taylor and Francis Group, USA, 2007, p. 62.
[10] Turton, J. F.; Kaufmann, M. E.; Warner, M.; Coelh, J.; Dijkshoorn, L.; van der Reiden, T. A prevalent, multiresistant clone of Acinetobacter baumannii in southeast England. J. Hosp. Infec., 2004, $58,170$.

[11] Satti, L.; Ikram, A.; Butt, T.; Malik, N.; Roshan, M. Multidrug resistant Acinetobacter species: an emerging superbug in hospital settings. Inf. Dis. J. Pak., 2009, 18, 44.

[12] Gad, G. F.; El-Domany, R. A.; Zaki, S.; Ashour, H. M. Characterization of Pseudomonas aeruginosa isolated from clinical and environmental samples in Minia, Egypt: prevalence, antibiogram and resistance mechanisms. J. Antimicrob. Chemother., 2007, 60, 2010.

[13] de Macedo, J. L.; Santos, J. B. Bacterial and fungal colonization of burn wounds. Mem. Inst. Oswaldo. Cruz., 2005, 100, 535.

[14] Patel, M. H.; Trivedi, G. R.; Patel, S. M.; Vegad, M. M. Antibiotic susceptibility pattern in urinary isolates of gram negative bacilli with special reference to AmpC $\beta$-lactamase in a tertiary care hospital. Urol Ann., 2010, $2,7$.

[15] Christenson, J. C.; Korgenski, E. K.; Daly, J. A. In vitro activity of meropenem, imipenem, cefepime and ceftazidime against Pseudomonas aeruginosa isolates from cystic fibrosis patients. $J$. Antimicrob. Chemother., 2000, 45, 899.

[16] Baines RD, Rifkind D. Intravenous administration of sodium colistimethate. JAMA, 1964, 190, 278.

[17] Pintado, V.; San Miguel, L. G.; Grill, F.; Mejia, B.; Cobo, J.; Fortún, J. Intravenous colistin sulphomethate sodium for therapy of infections due to multidrug-resistant Gram-negative bacteria. $J$. Infect., 2008, 56, 185.

Received: May 29, 2011

Revised: June 16, 2011

Accepted: June 22, 2011

(C) Satti et al.; Licensee Bentham Open.

This is an open access article licensed under the terms of the Creative Commons Attribution Non-Commercial License (http://creativecommons.org/licenses/by$\mathrm{nc} / 3.0 /$ ) which permits unrestricted, non-commercial use, distribution and reproduction in any medium, provided the work is properly cited. 